\title{
Flow Cytometry as a Tool to Study the Effects of Bacteriocins on Prokaryotic and Eukaryotic Cells
}

\author{
Harsh Mathur ${ }^{1,2 *}$, Mary C Rea ${ }^{2,3}$, Vincenzo Fallico², Paul D Cotter ${ }^{2,3}$, Colin Hill1,3 and Paul Ross $\mathbf{R}^{3,4}$ \\ ${ }^{1}$ School of Microbiology, University College Cork, Cork, Ireland \\ ${ }^{2}$ Teagasc Food Research Centre, Moorepark, Fermoy, County Cork, Ireland \\ ${ }^{3}$ APC Microbiome Institute, University College Cork, Cork, Ireland \\ ${ }^{4}$ College of Science, Engineering and Food Science, University College Cork, Cork, Ireland
}

\begin{abstract}
Researchers use a combination of techniques to study and contrast the impact of antimicrobials, such as bacteriocins, on sensitive and resistant variants. Flow cytometry is one such technique, which allows researchers to evaluate the activity of antimicrobials at a single-cell level in real-time. The generation of an increasing number of probes/dyes that can be used in flow cytometry studies has vastly expanded the potential applications of this technique. Furthermore, flow cytometry has the potential to replace, or at the very least be used as an adjunct to traditional growth-based techniques, including viable plate counts, growth curves, microscopic analysis and cell culture, many of which have limitations when used on their own. Here we review studies conducted using flow cytometry as a technique to assess the impact of antimicrobials from the bacteriocin family on individual cells, either prokaryotic or eukaryotic.
\end{abstract}

Keywords: Bacteriocins; Flow cytometry; Fluorochromes; Antimicrobials; Cell lines

\begin{abstract}
Abbreviations: CFU: Colony Forming Unit; DNA: Deoxyribonucleic Acid; PI: Propidium Iodide; RNA: Ribonucleic Acid; DiOC : 3,3'-Dipentyloxocarbocyanine Iodine; FACS: Fluorescence Activated Cell Sorting; OD: Optical Density; cFDA: Carboxyfluorescein Diacetate; CCCP: Carbonyl Cyanide $m$-Chlorophenyl Hydrazine; CFSE: Carboxyfluorescein Succinimidylester; $\mathrm{IC}_{50}$ : Half Maximal Inhibitory Concentration; SEM: Scanning Electron Microscopy; FITC: Fluorescein Isothiocyanate; PEA: Phosphatidylethanolamine; PlnA: Plantaricin A; HNSCC: Head and Neck Squamous Cell Carcinoma; ADP: Adenosine Diphosphate; PARP: Poly (ADP-ribose) Polymerase; STIs: Sexually Transmitted Infections; DiOC : 3,3' Dihexyloxacarbocyanine; NHE: $\mathrm{Na}^{+} / \mathrm{H}^{+}$Exchanger; HEK: Human Embryonic Kidney; VBNC: Viable but Non-culturable; LPS: Lipopolysaccharide; EDTA: Ethylenediaminetetraacetic; BCECF-AM: 2',7'-Bis(2-carboxyethyl)-5(6)Carboxyfluorescein Tetrakis(acetoxymethyl)Ester
\end{abstract}

\section{Introduction}

Bacteriocins are ribosomally synthesised antimicrobial peptides [1]. They are typically classified into two broad groups: class I (which are modified peptides) and class II (which are unmodified and/or cyclic peptides) [2]. Class I peptides can be further subdivided into several different subgroups, examples of which include lantibiotics (the most extensively studied subgroup), sactibiotics, labyrinthopeptins, thiopeptides and bottromycins [1]. Class II bacteriocins are typically divided into 5 subgroups (named class IIa-class IIe). Class IIa peptides include the pediocin PA1-type bacteriocins; class IIb are two-peptide unmodified bacteriocins; class IIc are circular peptides; class IId are linear non-pediocin-like single peptide bacteriocins and class IIe bacteriocins are peptides which undergo a non-ribosomal siderophoretype modification and are rich in serine residues in their C-termini $[1,2]$. Thus far, the vast majority of studies conducted to assess the antimicrobial properties of bacteriocins have involved standard growthbased techniques such as viable plate counts, deferred antagonism assays, kill curves, growth curves and/or microscopic analysis. There have already been quite a number of studies that have used flow cytometry as a tool to evaluate the antimicrobial actions of antibiotics. Such studies with antibiotics have generally involved assessing the permeability of cell membranes to different dyes, the presence or absence of metabolic activity and alterations in membrane potential [3-8]. However, to date, there have been relatively few such studies involving bacteriocins.

Recent studies have indicated that a routine overnight culture of isogenic bacteria is in fact composed of heterogenous sub-populations and these sub-populations behave in a different manner in response to alterations in the surrounding environment [9-11]. Techniques such as flow cytometry can help bridge the gap between our understandings of cell-cell interactions in an isogenic bacterial culture versus cell-cell interactions between different bacterial sub-populations in a culture. In a flow cytometer, cells or micrometric particles are passed through an interrogation point, at which point they are subjected to a laser beam. The light that the cell or particle absorbs, scatters or emits due to its physical properties is quantified. A flow cytometer is composed of fluidics, optics and electronic systems. The main purpose of the fluidics system is to allow the cells to pass through an interrogation point in a single file. The properties of a cell, such as its granularity, complexity and protein composition can be assessed based on light scattered at high angles (known as side scattering). In contrast, light scattered at low angles provides information about the size of the cell [12]. Cells are typically stained with fluorescent dyes to facilitate this task [13].

The study of individual cellular events can help us distinguish these events from an overall population response [14]. A particularly useful application of flow cytometry is the study of a bacterial response to a stimulus, such as exposure to antimicrobials. In a potential experimental situation involving antimicrobials, where there is no

*Corresponding author: Harsh Mathur, School of Microbiology, University College Cork, Cork, Ireland and Moorepark Food Research Centre, Teagasc, Fermoy, Cork, Ireland, Tel: +353 (0)21 4902745; E-mail: 105022894@umail.ucc. ie, harsh.mathur@teagasc.ie

Received December 09, 2015; Accepted January 23, 2016; Published January 25, 2016

Citation: Mathur H, Rea MC, Fallico V, Cotter PD, Hill C et al. (2016) Flow Cytometry as a Tool to Study the Effects of Bacteriocins on Prokaryotic and Eukaryotic Cells. J Mol Biomarkers Diagn S8:013. doi:10.4172/2155-9929.S8-013

Copyright: $\odot 2016$ Mathur $\mathrm{H}$, et al. This is an open-access article distributed under the terms of the Creative Commons Attribution License, which permits unrestricted use, distribution, and reproduction in any medium, provided the original author and source are credited 
difference found between a treated and untreated sample in terms of fluorescence emission subsequent to staining the cells with a dye, the possible causes may be a lack of a response to the stimulus, but may also be attributable to the response taking place in only a small percentage of cells. However, in order to definitively understand the apparent lack of a response, it is important to quantify the fluorescence emitted by each individual cell in the culture. Since flow cytometry assesses each cell individually, it provides real-time information regarding the impact of a stimulus on an overall population of cells. The parameters which are measured include integrity of the cell membrane with respect to permeability to dyes/stains, homeostasis of membrane potential, as well as disruption/aberration of metabolic activity inside the cell, as assessed by quantifying the magnitude of a fluorescent signal. This occurs by means of a non-fluorescent substrate being enzymatically converted to a fluorescent metabolite [3]. A key advantage of flow cytometry is that it is equally suitable to study bacteriostatic or bactericidal effects of antimicrobials such as antibiotics or bacteriocins. On the other hand, colony forming unit (CFU) counts merely reveal the number of cells which are healthy enough to form colonies on agar plates. Flow cytometry in contrast, can provide information regarding the percentage of cells in a population which are damaged or stressed but still metabolically active. Such types of cells may not be able to form colonies, even upon transfer to rich media [4,15-18].

The advancement of flow cytometry along with the introduction of novel fluorochromes/probes allows us to study the viability of cells, membrane structure, integrity, and membrane potential at a single-cell level. This has opened up an avenue of possibilities for the assessment of susceptibility of bacterial strains to bacteriocins. With the help of fluorescence probes, flow cytometry has the ability to measure the fluorescence intensity of cell contents as well as metabolic activity. Thus, it adds an extra layer of information with respect to alterations in cell size, morphology and fluorescence $[4,18,19]$. The probes which are typically used in flow cytometry studies, can be divided into two broad groups: i) nucleic acid binding dyes and ii) metabolic/cellular/ protein binding dyes, examples of which include dyes to measure membrane potential [4]. DNA staining dyes include mithramycin with ethidium bromide, as well as propidium iodide (PI) $[4,20]$. Since PI is a DNA-intercalating agent, it is most commonly used to discriminate within a mixture of live/dead cells [4]. However, PI is not taken up by live cells and therefore when used on its own, merely permits the detection and quantification of dead cells. Examples of other nucleic acid-based fluorochromes include Syto 13 and Syto 17, both of which can bind DNA and RNA [21]. A further useful nucleic acid binding fluorochrome is acridine orange, which has the ability to stain both DNA and RNA with different wavelength emissions [22]. Since the integrity of the cell membrane affects the influx of acridine orange into the cells, this fluorochrome can be utilised to assess membrane damage elicited by an antimicrobial, such as a bacteriocin. Typically, bacteria react to antimicrobials, including bacteriocins, by either increasing or decreasing their membrane potential and examples of probes used to measure membrane potential are 3,3'-dipentyloxocarbocyanine iodine $\left(\mathrm{DiOC}_{5}\right)$, bis(1,3-dibutylbarbituric acid)trimethine oxonol and rhodamine 123 [23-25].

In this review, we describe some studies that use flow cytometry as a tool to evaluate the effects that bacteriocins have on individual cells, both prokaryotic and eukaryotic cell lines. More specifically, in the context of these studies, we focus on the advantages, disadvantages and possible future applications of flow cytometry as an approach to complement traditional growth-based/cell culture based-assays.

\section{Use of flow cytometry to study the effects of bacteriocins on prokaryotic cells}

In a recent study, Chopra et al. described the discovery of a novel bacteriocin, sonorensin, which possesses potent antimicrobial activity against biofilms [26]. This peptide belongs to the newly designated heterocycloanthacin subclass of bacteriocins. Flow cytometry was used to demonstrate the damage caused to the membrane of sensitive Staphylococcus aureus cells through exposure to sonorensin or the prototypical bacteriocin, nisin (a member of the lantibiotic class of bacteriocins), using the stain PI. The results showed that membrane integrity in S. aureus cells was compromised subsequent to bacteriocin exposure, as measured by the increases in PI fluorescence, with $70.0 \%$ fluorescence intensity quantified with the stain PI due to sonorensin exposure (indicating that $70 \%$ of the cells were dead), and $68.1 \%$ fluorescence intensity with the same probe, subsequent to nisin exposure (indicating that $68.1 \%$ of the cells were dead) [26]. Overall, the study helped to provide initial insights into the mechanism of action of the novel bacteriocin sonorensin, showing that the peptide elicited permeabilization of the $S$. aureus cell membrane [26].

There have been a few studies conducted investigating the antimicrobial effects of nisin used independently and in combination with other antimicrobials. With respect to the antimicrobial effects of nisin used independently, Weeks et al. studied the effects of the peptide on the food pathogen Listeria monocytogenes ScottA using fluorescence activated cell sorting (FACS) [27]. FACS is a similar technique to flow cytometry, with the added advantage of enabling researchers to 'sort' or separate different sub-populations of cells for downstream molecular/ physiological assessment [28]. With the use of the probe PI, Weeks and co-workers found that membrane integrity was compromised and that the sensitivity of the strain to nisin was dependent on the growth phase, with $\log$ phase cells at an optical density $\left(\mathrm{OD}_{600}\right)$ of 0.38 being considerably more sensitive to the peptide than stationary phase cells with an $\mathrm{OD}_{600}$ of 1.20 . The use of FACS in the study helped to reveal the alterations taking place in the membrane, governing susceptibility to nisin [27]. In a separate study by Budde and Rasch, the authors used both viable plate counts $(\mathrm{CFU} / \mathrm{ml})$ and flow cytometric analysis to evaluate the antimicrobial actions of the bacteriocins nisin, pediocin PA1 and sakacin A (the latter two being class IIa bacteriocins) on the target strains Lactobacillus sakei NCFB 2714, Lactobacillus reuteri DSM 12246 and $L$ b. sakei DSM 20017 with a view to determining if there is a correlation between the two methods [29]. The stain carboxyfluorescein diacetate (cFDA), which measures esterase activity intracellularly, was used to determine the fluorescence intensity (FI) of the cells and was compared to CFU/ml counts. Since cFDA is cleaved by esterases inside the cell with the consequent release of the fluorescent substance carboxyfluorecein, it is a useful fluorochrome for determining viable cell numbers [30,31]. While a general pattern of decreased FI from the cells following exposure to bacteriocins was observed across the board, the reductions in FI and decrease in $\mathrm{CFU} / \mathrm{ml}$ counts at corresponding bacteriostatic concentrations were not consistent with one another [29]. Overall, the measurement of FI turned out to be the more sensitive method of evaluating antimicrobial effects as a consequence of bacteriocin exposure. Indeed, the leakage of cFDA from cells exposed to pediocin PA1 led to a reduction in FI, whereas these damaged cells could still be detected as colony forming units upon transfer to rich growth media [29]. This repair of damaged cells upon transfer to rich media was less apparent in sakacin A-exposed and nisin-exposed cells. In the same study, the authors showed using both flow cytometry and $\mathrm{CFU} / \mathrm{ml}$ counts that the antibacterial activity of pediocin PA1 was ameliorated at elevated temperatures [29]. 
Some studies have also explored the effects of nisin in combination with other stressors using flow cytometry. For instance, Ayari et al. reported the impact that nisin has, when combined with repetitive sub-lethal radiation processing, on Bacillus cereus cells [32]. Flow cytometry in the study was able to show that $B$. cereus cells exposed to nisin were more likely to develop radio-resistance when exposed to repeated sub-lethal doses of $\gamma$-irradiation, compared to cells which were not exposed to nisin. This increased resistance to $\gamma$-irradiation was not demonstrated with standard viable plate counts independently, thus highlighting the value of using flow cytometry as an adjunct to viable plate counts. In addition, flow cytometry in the study was also used to show the physiological alterations that took place in B. cereus cells whilst developing resistance to $\gamma$-irradiation [32]. In a separate study, Dalmau and co-workers investigated the combined activities of lactacin F (a class IIb bacteriocin) and nisin and demonstrated that, when combined, these two bacteriocins behave in an additive synergistic fashion [33]. The bactericidal effects of the two bacteriocins were studied using flow cytometry. While it was shown that the extent of damage caused to Lactobacillus helveticus cells by nisin or lactacin $\mathrm{F}$ was both concentration- and time-dependent, the injuries elicited by lactacin $\mathrm{F}$ or nisin in Lb. helveticus membranes caused slightly different flow cytometric profiles. At low nisin concentrations, there was an influx of PI into Lb. helveticus cells, with the majority of cells losing the fluorochrome Syto 9 with exposure to the peptide for a longer duration of time. Syto 9 is a green fluorescent nucleic acid staining probe which can permeate the cell membrane and is a component of the BacLight Bacterial Viability kits along with PI, and is commonly used in flow cytometry studies. In contrast, with lactacin $\mathrm{F}$, the flow cytometric profiles showed that Syto 9 was lost from the cells soon after exposure to the peptide, with an incorporation of PI largely proportionate to the duration of exposure to the peptide [33]. Since nisin initially led to an increase in PI, it suggests the formation of wide channels as a consequence of exposure. As Syto 9 failed to enter the cells subsequent to a short exposure to lactacin F, it is likely that the peptide caused a sharp decrease in membrane potential. When lactacin $\mathrm{F}$ and nisin were combined, flow cytometry revealed a mixture of the effects seen with nisin or lactacin $\mathrm{F}$ alone, presumably because a certain percentage of cells were affected by nisin, whereas the rest were affected by lactacin F [33]. It is likely that each of the bacteriocins behaves separately but the additive effects observed might be a result of a rise in the number of single membrane injuries in the sensitive indicator [33]. The protonophore carbonyl cyanide $m$-chlorophenyl hydrazine (CCCP) was also used in flow cytometry experiments in the same study to assess the importance of the impact on a cell's proton motive force, with respect to the antimicrobial mode of action of nisin and lactacin F [33]. Low levels of CCCP used independently in control cultures resulted in similar profiles to those obtained with the addition of lactacin F alone. Surprisingly, however, higher levels of CCCP added independently to the control cultures failed to elicit the influx of PI into the cells. The exposure of cells to lactacin $\mathrm{F}$ for a longer time, with a simultaneous addition of CCCP however, resulted in the incorporation of PI into the cells, suggesting the formation of a few lactacin F channels at either low membrane potential or when the membrane potential was zero [33]. Thus, caution must be exercised when interpreting flow cytometry profiles using CCCP, as it is difficult to ascertain whether any alterations in profiles are triggered by the bacteriocin peptide(s), triggered by CCCP or a combination thereof. In contrast to lactacin $\mathrm{F}$, the addition of nisin together with CCCP resulted in an unexpected decrease in the percentage of dead cells from $23.8 \%$ to $8.5 \%$ after 10 minutes of exposure to the peptide. This shift in the population of dead cells was again difficult to interpret due to the unpredictable effects elicited by CCCP. Importantly in the study however, flow cytometry profiles indicated additive effects against sensitive L $b$. helveticus cells, when both nisin and lactacin $\mathrm{F}$ were added together [33]. Ueckert et al. also used a combination of viable plate counts, fluorometry and flow cytometry to assess the combined effects of nisin and heat treatment on sensitive Lactobacillus plantarum cells [34]. The study showed that this combination had synergistic effects against the target cells. More specifically, synergistic effects were seen when $\log$ phase $\mathrm{Lb}$. plantarum cells were initially exposed to heat between $48-54^{\circ} \mathrm{C}$, followed by treatment with nisin at concentrations up to $0.5 \mu \mathrm{g} / \mathrm{ml}$. Interestingly, addition of nisin followed by heat treatment was not as effective in reducing $L b$. plantarum viability. As the antimicrobial activity of nisin is dependent on membrane potential, the growth phases of target cells can lead to differences in sensitivity to the peptide. Essentially, log phase cells were found to be more susceptible to nisin and heat treatment relative to stationary phase cells in the study [34]. Any alterations in membrane permeability of sensitive $L b$. plantarum cells were measured using the dye PI, whereas esterase activity was assessed using cFDA fluorescence. As expected, sub-populations responding differently to heat and nisin treatment were apparent. For example, heat treatment at $48^{\circ} \mathrm{C}$ alone for 5 minutes resulted in only $0.27 \%$ of total $L b$. plantarum cells having membrane damage, while the majority of cells displayed high esterase activity (as quantified by cFDA fluorescence). In contrast, exposure of Lb. plantarum cells to nisin on its own resulted in a high percentage of cells with membrane damage (as quantified by a high PI fluorescence measurement). Overall, the numbers of membrane-damaged cells were increased in samples treated with heat followed by nisin, as a result of the synergistic effects of the two treatments [34]. A separate study by Ueckert and co-workers described the use of flow cytometry as a tool to study aspects of the lag phase of growth, cell division and injury to $L b$. plantarum cells following heat stress and exposure to nisin. In this case, the fluorescent dyes carboxyfluorescein succinimidylester (CFSE) and PI gave insights into the extent of damage caused by stress and assisted in the differentiation of sub-populations in the bacterial culture as a result of stress [35]. CFSE is a useful marker for assessing cell division as it has the ability to permeate the cell membrane and to bind aliphatic amine residues inside the bacterial cell [36].

With respect to other bacteriocins and their targeting of Grampositive organisms, Martínez et al. also used flow cytometry, with the aid of the stain PI, to study the impact that the class IId bacteriocin lactococcin 972 has on the morphology and viability of the target strain Lactococcus lactis MG1614 [17]. Overall, flow cytometry did not show any significant alterations in the morphology of sensitive $L$. lactis cells during the first hour subsequent to lactococcin 972 exposure. In contrast, phase-contrast microscopy was able to detect significant changes in the morphology of these treated cells during the first hour, thus highlighting the value of using a combination of techniques to evaluate the antimicrobial effects of bacteriocins [17]. In another study, Swarts et al. used a variety of methods including flow cytometry, OD measurements and viable plate counts to assess the growth profile of $L$. monocytogenes exposed to the class IIa bacteriocin, leucocin B-TA11a [37]. With a view to measuring the sensitivity of the BacLight system, live and dead cultures were combined such that either the live cells population or the dead cells population was greater than the other. As controls, live and dead cells were mixed in increments between $10 \%$ $100 \%$. The BacLight staining procedure was able to distinguish between live and dead cells in mixed cultures in ratios between 10-100\% live or dead cells, as high correlations $\left(r^{2}=0.97\right.$ and $\left.r^{2}=0.99\right)$ were obtained between relative cell numbers and relative proportions of live or dead cells between these ratios of $10-100 \%$. However, relatively poor 
correlations were obtained for populations containing less than $10 \%$ live or dead cells in the mixture with values of $\mathrm{r}^{2}=0.72$ and $\mathrm{r}^{2}=0.20$ respectively. The influx of BacLight dyes into L. monocytogenes cells treated with leucocin B-TA11a indicated that the cells were viable but the membrane was compromised, most likely due to pore formation caused by the bacteriocin. Thus, while it was shown that leucocin B-TA11a appeared to initially inhibit L. monocytogenes, flow cytometric analysis using BacLight dyes indicated that the cells were viable but leaky [37].

Finally, the antibacterial effects of the class IIb bacteriocins, plantaricin E/F and plantaricin J/K on a target Escherichia coli strain were reported in a recent study [38]. Gram negative strains are usually resistant to bacteriocins produced by Gram positive bacteria due to the presence of an outer membrane, but the authors demonstrated that a compromised outer membrane rendered E. coli $\mathrm{K} 12$ cells sensitive to plantaricins. To this end, the sensitivity of an outer membrane lipoprotein-defective lpp mutant of $\mathrm{K} 12$ to plantaricin was studied. The authors used live/dead stains as part of their flow cytometry experiments. As expected, a decrease in cell viability was observed in the $l p p$ mutant, relative to the wild type strain, using both live/dead staining assessed with flow cytometry, as well as in terms of $\mathrm{CFU} / \mathrm{ml}$ counts [38].

A summary of the use of flow cytometry to evaluate the antimicrobial effects of bacteriocins on either bacterial cells or eukaryotic cell lines, including the fluorochromes used in the studies (Table 1).

\section{Use of flow cytometry to study the effects of bacteriocins on eukaryotic cells}

A recent study by Chen et al. reported the effects of the modified antimicrobial peptide KL15 on colon adenocarcinoma cell lines [39]. KL15 is a modified bacteriocin based on the sequences of the bacteriocins $\mathrm{m} 2386$ and $\mathrm{m} 2163$. The bacteriocin $\mathrm{m} 2163$ belongs to the class IId subgroup of bacteriocins, whereas $\mathrm{m} 2386$ has yet to be classified on account of its $\mathrm{ABC}$ transporter and histidine kinase gene sequences being incomplete [40]. KL15 has the ability to inhibit adenocarcinoma cells Caco-2 and SW480 with half maximal inhibitory concentration $\left(\mathrm{IC}_{50}\right)$ values of $50 \mu \mathrm{g} / \mathrm{ml}$. In contrast, the $\mathrm{KL} 15 \mathrm{IC}_{50}$ value for killing the healthy human mammary epithelial cells H184B5F5/M10 is $150 \mu \mathrm{g} / \mathrm{ml}$. The authors were able to show increased cell membrane permeability among SW480 cells treated with KL15 using a combination of flow cytometry and confocal microscopy. The use of scanning electron microscopy (SEM) confirmed the formation of pores in the membrane of these cells, caused by KL15 [39]. Briefly, the authors analysed the effects of KL15 on the cell cycle of SW480 cells by using the dye PI, and by using this probe, cells in the G1, S and G2/M phase were identified [39]. The G1 phase represents the first phase of the cell division in eukaryotic cells, in which mRNA and proteins are synthesised. This G1 phase is followed by the S and G2 phases (all together comprising interphase), followed by the final step of cell division which involves mitosis ( $M$ phase) $[40,41]$. Chen and co-workers found that as the KL15 exposure time increased from 1 minute to 24 hours, there was a corresponding elevation in the percentage of sub-G1 populations from $1.49 \%$ to $5.05 \%$ [39]. With increasing concentrations of KL15, from $40-120 \mu \mathrm{g} / \mathrm{ml}$, there was a consequent increase in the sub-G1 population from $3.74 \%$ to $4.40 \%$, with cells exposed to the bacteriocin for 24 hours. Using flow cytometry with the aid of the dyes PI and AnnexinV-fluorescein isothiocyanate (FITC), the authors were able to differentiate between early apoptosis, late (apoptotic-necrotic) cells and primary necrotic cells, as a consequence of cell damage caused by KL15 exposure [39]. Annexin V-FITC is an extremely useful fluorochrome as it binds phosphatidylserine (a marker of the early stages of apoptosis) [40-42]. As expected, there was a correlation between increases in KL15 concentrations and increases in percentages of late apoptotic/necrotic cells in the study [40]. Interestingly, there was no significant difference found between cells exposed to $40 \mu \mathrm{g} / \mathrm{ml} \mathrm{KL} 15$ and the control. In contrast, there were significant shifts in PI fluorescence intensities at KL15 concentrations of $80-120 \mu \mathrm{g} / \mathrm{ml}$, indicating that the cell membrane was damaged as a result of the KL15 peptide and that the effects were dose-dependent [39].

Yates and co-workers studied the anti-proliferative characteristics of the lantibiotic duramycin in tumor cells, as well as its effects on the viability of such tumor cells [43]. Aside from its antimicrobial activity, duramycin also causes perturbations in tumor cell surface-based coagulation processes. Since duramycin targets phosphatidylethanolamine (PEA) on the surface of cells, flow cytometry was used to detect PEA on the surface of cell lines exposed to this bacteriocin. Indeed, duramycin was used as a 'ligand' in order to label PEA on the surface of pancreatic tumor cells and it was found that PEA expression was elevated in apoptotic cells and in particular, necrotic cells [43]. Flow cytometry in the study helped to reveal that an increase in apoptosis and necrosis of tumor cells was duramycin dose-dependent and that a decrease in tumor cell proliferation was also dependent on the concentration of the peptide [43]. In a separate study, the effects of the class IIb bacteriocin, plantaricin A (PlnA) on the membranes of normal and cancerous lymphocytes as well as neuronal cells were reported by Sand and co-workers [44]. The authors assessed if PlnA had a greater impact on cancerous cells than normal neuronal cells and lymphocytes. Flow cytometry was used to assess morphological alterations (scatter) and viability (uptake of the dead stain PI) in the normal human B and $\mathrm{T}$ lymphocytes, Jurkat cells and Reh cells. The technique highlighted that exposure of lymphocytes to PlnA elicited an increase in side scatter compounded by a decrease in forward scatter, pointing towards reduced particle size and an increase in granularity, with more dramatic effects observed with increased PlnA concentrations [44]. The damage caused to the membranes of Reh cells, B and T lymphocytes, as detected by alterations in morphology measured by changes in forward and side scatter using flow cytometry was PlnA dose-dependent. Similarly, decreases in cell viability of lympohocytes caused by PlnA were also concentration-dependent. Importantly, however, the study did reveal that normal $\mathrm{T}$ and $\mathrm{B}$ lymphocytes weren't affected as much as the cancerous Jurkat and Reh cell lines at PlnA concentrations between 10$50 \mu \mathrm{M}$ [44]. With these flow cytometry-based experiments, together with measurements of membrane conductance and $\mathrm{Ca}^{2+}$ imaging, it was determined that overall, PlnA has a largely similar effect on both normal and cancerous neuronal cells and lymphocytes, and does not have preferential effects on cancerous cells [44]. Interestingly, in contrast to this more recent study by Sand et al., in a separate study by the same group, cancerous cells were found to be more sensitive to PlnA than normal rat anterior pituitary cells $[44,45]$.

On the basis of the work conducted by Joo et al., who showed that nisin at concentrations of $2.5 \%$ possesses anti-tumor effects against head and neck squamous cell carcinoma (HNSCC) both in in vitro and in vivo experiments, Kamarajan and co-workers investigated the potential anti-cancer effects of a natural variant of nisin, named nisinZP in a recent study [46,47]. Flow cytometry, with the use of the stain Annexin $\mathrm{V}$ was used to evaluate the apoptotic effects of three different variants of nisin on HNSCC cells in the study [47]. Using this stain and flow cytometry, it was found that apoptosis of HNSCC cell lines elicited by nisin involves the cleavage of caspase- 8 and poly (ADP-ribose)- 
Citation: Mathur H, Rea MC, Fallico V, Cotter PD, Hill C et al. (2016) Flow Cytometry as a Tool to Study the Effects of Bacteriocins on Prokaryotic and Eukaryotic Cells. J Mol Biomarkers Diagn S8:013. doi:10.4172/2155-9929.S8-013

Page 5 of 6

\begin{tabular}{|c|c|c|c|}
\hline Bacteriocin(S) & Target Bacterial Species & Fluorochromes used & References \\
\hline Sonorensin, nisin & S. aureus & $\mathrm{PI}$ & [26] \\
\hline Lactacin F, nisin & Lb. helveticus & Syto 9/PI & [33] \\
\hline Nisin & L. monocytogenes ScottA & PI & [27] \\
\hline Pediocin PA1, sakacinA, nisin & $\begin{array}{l}\text { Lb. sakei NCFB2714, Lb. sakei DSM20017, Lb. reuteri } \\
\text { DSM12246 }\end{array}$ & cFDA & [29] \\
\hline Nisin with sub-lethal radiation processing & B. cereus & cFDA and $\mathrm{PI}$ & [32] \\
\hline Nisin and heat treatment & Lb. plantarum & cFDA, Ethidium Bromide, PI & [34] \\
\hline Nisin & Lb. plantarum & 5-and 6-CFSE, PI & [35] \\
\hline $\begin{array}{l}\text { Nisin subsequent to high pressure } \\
\text { processing }\end{array}$ & L. monocytogenes, S. Typhimurium & Syto 9/PI & {$[80]$} \\
\hline Plantaricin E/F, plantaricin J/K & E. coli & Syto 9/PI & [38] \\
\hline Leucocin B-TA11a & L. monocytogenes & $\mathrm{PI}$ & [37] \\
\hline Lactococcin 972 & L. lactis MG1614 & $\mathrm{PI}$ & [17] \\
\hline Mesentericin Y105 & Listeria spp. & $\mathrm{DiOC}_{6}$ & [81] \\
\hline Bacteriocin(S) & Eukarytotic cell lines & Fluorochromes used & References \\
\hline KL15 & Colon adenocarcinoma cell lines & PI, Annexin V-FITC & [39] \\
\hline NisinZP & Head and neck squamous cell carcinoma & Annexin V & [47] \\
\hline Duramycin & Tumor cell lines & BacLight kit & [43] \\
\hline Nisin & Sperm & $\mathrm{PI}$ & [48] \\
\hline Plantaricin A & B and T lymphocytes, Jurkat cells, Reh cells & $\mathrm{PI}$ & [44] \\
\hline Halocin H6 & NIH3T3, HL1, Jurkat, myocytes, fibroblasts & BCECF-AM & [51] \\
\hline Microcin E492 & HeLa cells & Annexin V-FITC, $\mathrm{DiOC}_{6}, \mathrm{PI}$ & [49] \\
\hline Colicins E1, E3, A and U & Fibroblasts and 11 tumor cell lines & $\mathrm{PI}$ & {$[50]$} \\
\hline
\end{tabular}

Table 1: Effects of bacteriocins on bacterial cells or eukaryotic cell lines using flow cytometry.

polymerase (PARP), as well as involving inactivation of calpain 1 [47]. A separate study by Gupta et al. was also conducted to assess the impact of nisin on cell membranes and its suitability as a vaginal microbicide [48]. Flow cytometry was utilised to quantify the amount of membrane damage elicited by the peptide and further confirmed by SEM. Using the stain PI, flow cytometry revealed that nisin selectively permeabilizes the membranes of bacteria and sperm and these findings corroborated ultra-structural alterations described in the study. The use of liposome model vesicles also revealed that calcein leakage from liposomes from bacteria and sperm was caused by nisin, whereas in contrast, red blood corpuscles were not affected by the peptide [48]. Thus, nisin could be harnessed to utilise the peptide as a vaginal microbicide to prevent sexually transmitted infections (STIs) and as a contraceptive.

With respect to bacteriocins produced by Gram negatives, the effects of microcin E492 (produced by a Klebsiella pneumoniae strain) on HeLa cells was assessed in a study [49]. Flow cytometry in combination with the stain Annexin V-FITC was predominantly used in the study. The stain 3, 3' dihexyloxacarbocyanine ( $\mathrm{DiOC}_{6}$ ) was also used to quantify the loss of membrane potential from mitochondria of HeLa cells following exposure to the peptide. In addition, flow cytometer helped to reveal that cell death and fragmentation of DNA was dependent on the concentration of microcin E492 and populations of HeLa cells corresponding to M0 (viable cells), M1 and M2 (both permeable to $\mathrm{PI}$ ) were distinguished using this technique. The effects of microcin E492 on the light scattering characteristics of HeLa cells were also evaluated using side scatter versus forward scatter plots in the study [49]. In a separate study, flow cytometry was used to evaluate the effects of the pore-forming Gram-negative bacteriocins, colicins A, U, E1 and E3, on a fibroblast cell line as well as the effects on 11 tumor cell lines which had mutations in their $p 53$ genes [50]. While it was shown that colicin A prevented the growth of all the cell lines tested in the study, colicin $U$ and colicin E3 did not inhibit the growth of these tumor cell lines. Colicin E1 prevented the growth of all the cell lines, bar one, that were evaluated in the study. The growth inhibition effects of colicin A on different cell lines ranged from $16-56 \%$, while the growth rate of the standard fibroblast cell line MRC5 decreased by $36 \%$ due to colicin A exposure. In contrast, the growth inhibition rates of colicin $\mathrm{E} 1$ on different tumor cell lines ranged from $17-40 \%$, though the inhibition rates against the standard fibroblast cell line were $11 \%$. Significantly, flow cytometry was able to show that colicin E1 and colicin A triggered a 12-58\% increase in the number of cells in the G1 phase of growth and in the apoptotic phase by $7-58 \%$, in three of the cell lines used in the study [50].

Finally, flow cytometry was also used to assess the impact the bacteriocin halocin $\mathrm{H} 6$ has on the $\mathrm{Na}^{+} / \mathrm{H}^{+}$exchanger (NHE) in mammalian cells in another study [51]. Halocin H6 is produced by Haloferax gibbonsii $\mathrm{SH} 7$, a member of the haloarchaea group. This bacteriocin-like protein is $32 \mathrm{kDa}$ in size and its mechanism of action involves blocking the $\mathrm{Na}^{+} / \mathrm{H}^{+}$ antiporter in susceptible bacterial strains $[52,53]$. It was found that NHE activity was inhibited by halocin $\mathrm{H} 6$ in mammalian cells lines such as NIH3T3, HEK293, HL1 and Jurkat, as well as in myocytes and fibroblasts and this effect was concentration-dependent. Interestingly, halocin H6 is the first biological compound which has been reported to inhibit NHE activity in eukaryotic cells [51].

A summary of the use of flow cytometry to evaluate the effects of bacteriocins on eukaryotic cells is included in Table 1.

\section{Other Applications of Flow Cytometry, Limitations and Conclusions}

Apart from being able to assess the sensitivity of bacterial strains to different antimicrobials, flow cytometry has a myriad of applications with respect to the elucidation of the mechanism of action of such antimicrobial drugs, as well as in the study of cell-cell interactions, quorum sensing, sporulation, formation of biofilms, diagnosis of 
infections, identification of microbes and detection of serum antibodies. Due to the capability of detecting heterogeneous populations and detection of numerous aspects of cell function, flow cytometry can be used to detect polymicrobial infections or the impact of treating pathogens with antibiotics [4,25,54-63]. Many of these goals are difficult to achieve with culture-based techniques on their own. Fluorescent probes in conjunction with flow cytometry have the capability of detecting alterations in cells such as aberrations in permeabilization and membrane potential [3,64-66]. As several different parameters can be assessed by flow cytometry at a single-cell level, its use has the potential to cause a paradigm shift and may be considered a more accurate method of assessing cell function than traditional cell viability and cell growth-based methods, in future studies. In this context, it must be emphasised that every bacterial strain reacts in a different manner to different drugs and, thus, no unified formula can be used to determine if correlations exist between cytometric and viability plate count assays. What is certain is that flow cytometry is an extremely accurate and sensitive method, with data obtained in real-time, and is less time consuming than growth-based techniques for studying the impact of antimicrobials on bacterial strains $[4,62]$. The discrepancies frequently observed between growth-based methods and cytometricbased methods can be attributed to the presence of metabolically active but non-cultivable cells (VBNC) $[67,68]$. Worryingly, VBNC have been detected from a range of clinically relevant and supposedly sanitary source in situations whereby the isolation and growth of viable cells is not appropriate, using cytometric techniques $[69,70]$.

The study of sub-populations within a bacterial culture and analysis of cells at a single-cell level also has the potential to enhance our conception of the responses of bacterial cells to bacteriocins, and indeed other stressors. Any questions regarding differences in fluorescence intensity observed in cells subsequent to a challenge by bacteriocins can only be answered by studying the fluorescence emission profiles of each individual cell in the culture by using techniques such as flow cytometry. Apart from permitting the detection of heterogeneity of responses to an antimicrobial within a sub-population of cells, another key advantage of flow cytometry which must not be under-estimated, is that it can detect resistant variants within a given sub-population and this is particularly relevant for clinical/medical applications with respect to antimicrobials/bacteriocins [19].

Despite its numerous benefits, scientists should still be aware that flow cytometry also has potential drawbacks. A possible limitation of using certain stains is that permeabilization caused by certain dyes can be toxic to some cells [4]. Another limitation is the difficulty in distinguishing small fragmented cells and background particles/ debris, which can be resolved by using a combination of forward scatter and analysis of the fluorescence signals generated [12,54]. In addition, the use of nucleic acid stains should circumvent this problem as such stains only target cells and not particles/debris. Another problem is that certain types of bacterial cells in particular, tend to aggregate or cluster, which precludes their analysis at a singlecell level. Therefore, samples must be homogenized and disaggregated prior to flow cytometric analysis [71-73]. A further limitation includes the variations in the ability of different bacterial strains to take up membrane potential stains [74]. For example, rhodamine 123 stains Gram-positive and Gram-negative strains differently due to the charge on lipopolysaccharide (LPS) present on the surface of Gram-negatives [75]. Indeed, Gram-negative cells are frequently treated with chelating agents such as ethylenediaminetetraacetic acid (EDTA) to allow dyes such as rhodamine 123 to enter the cells. An important consideration in these types of experiments is that such pre-treatments can affect the viability and membrane potential of the cells $[75,76]$. Furthermore, variations amongst strains with respect to the efflux of internalised stains may result in differences in results [77-79]. In addition, the length of time that the cells are stained for, as well as the concentration of the dyes may have an impact on flow cytometric profiles $[16,23,59]$. Finally, the growth phase of the bacterial cells is an important factor when conducting flow cytometry studies [80-81]. Stationary phase cells are stable for a longer time than log or dead phase cells and stationary phase cells are most commonly used in traditional antimicrobial assays as well [23].

Despite these issues, flow cytometry has a plethora of applications in the medical, biotechnology and research fields and the increase in the number of probes available further augments the number of applications of this technique. Indeed, the use of personalised probes and dyes for the detection of changes in specific targets and intracellular activities permits the targeted use of flow cytometry to ascertain the structural and functional characteristics of a population of cells.

\section{Acknowledgements}

$\mathrm{HM}$ is a researcher in Teagasc Food Research Centre and the School of Microbiology, University College Cork, funded by the Science Foundation of Ireland (SFI)-funded Centre for Science, Engineering and Technology and the Alimentary Pharmabiotic Centre Microbiome Institute (APC) Grant Number SFI/12/ $\mathrm{RC} / 2273$. Research in PDC, $\mathrm{CH}, \mathrm{MRC}, \mathrm{VF}$ and RPR laboratories is supported by the Science Foundation of Ireland (SFI)-funded Centre for Science, Engineering and Technology and the APC Microbiome Institute (APC).

\section{References}

1. Cotter PD, Ross RP, Hill C (2013) Bacteriocins - a viable alternative to antibiotics? Nat Rev Microbiol 11: 95-105

2. Rea MC, Ross RP, Cotter PD, Hill C (2011) Classification of bacteriocins from Gram-positive bacteria. Prokaryotic Antimicrobial Peptides: From Genes to Applications. Springer Publishing Inc. New York, NY, USA.

3. Shapiro HM (2000) Microbial analysis at the single-cell level: tasks and techniques. J Microbiol Methods 42: 3-16.

4. Walberg M, Gaustad P, Steen HB (1997) Rapid assessment of ceftazidime ciprofloxacin, and gentamicin susceptibility in exponentially-growing $\mathrm{E}$. coli cells by means of flow cytometry. Cytometry $27: 169-178$

5. Walberg M, Gaustad P, Steen HB (1996) Rapid flow cytometric assessment of mecillinam and ampicillin bacterial susceptibility. J Antimicrob Chemother 37 1063-1075.

6. Dessus-Babus S, Belloc F, Bébéar CM, Poutiers F, Lacombe F, et al. (1998) Antibiotic susceptibility testing for Chlamydia trachomatis using flow cytometry Cytometry 31: 37-44

7. Assunção P, Antunes NT, Rosales RS, Poveda C, de la Fe C, et al. (2007) Application of flow cytometry for the determination of minimal inhibitory concentration of several antibacterial agents on Mycoplasma hyopneumoniae. J Appl Microbiol 102: 1132-1137.

8. Soejima T, Minami J, Iwatsuki K (2012) The exclusive use of flow cytometry to evaluate the antibiotic-susceptibility. Biochim Biophys Acta 1820: 1980-1986.

9. Rainey PB, Beaumont HJ, Ferguson GC, Gallie J, Kost C, et al. (2011) The evolutionary emergence of stochastic phenotype switching in bacteria. Microb Cell Fact 10 Suppl 1: S14.

10. Fraser D, Kaern M (2009) A chance at survival: gene expression noise and phenotypic diversification strategies. Mol Microbiol 71: 1333-1340.

11. de Jong IG, Veening J, Kuipers OP (2012) Single cell analysis of gene expression patterns during carbon starvation in Bacillus subtilis reveals large phenotypic variation. Environ Microbiol 14: 3110-3121.

12. Leif RC (1995) Practical Flow Cytometry. (edn $3^{\text {rd }}$ ) Wiley-Liss, Inc; New York, NY, USA.

13. Veal DA, Deere D, Ferrari B, Piper J, Attfield PV (2000) Fluorescence staining and flow cytometry for monitoring microbial cells. J Immunol Methods 243: 191-210.

14. Müller S, Davey H (2009) Recent advances in the analysis of individua 
Citation: Mathur H, Rea MC, Fallico V, Cotter PD, Hill C et al. (2016) Flow Cytometry as a Tool to Study the Effects of Bacteriocins on Prokaryotic and Eukaryotic Cells. J Mol Biomarkers Diagn S8:013. doi:10.4172/2155-9929.S8-013

microbial cells. Cytometry A 275: 83-85.

15. Durodie J, Coleman K, Simpson IN, Loughborough SH, Winstanley DW (1995) Rapid detection of antimicrobial activity using flow cytometry. Cytometry 21 : 374-377.

16. Gant VA, Warnes G, Phillips I, Savidge GF (1993) The application of flow cytometry to the study of bacterial responses to antibiotics. J Med Microbiol 39: $147-154$

17. Martínez B, Rodríguez A, Suárez JE (2000) Lactococcin 972 , a bacteriocin that inhibits septum formation in lactococci. Microbiology $146: 949-955$.

18. Pore RS (1994) Antibiotic susceptibility testing by flow cytometry. J Antimicrob Chemother 34: 613-627.

19. Walberg M, Gaustad P, Steen HB (1997) Rapid discrimination of bacteria species with different ampicillin susceptibility levels by means of flow cytometry. Cytometry 29: 267-272.

20. Steen HB, Boye E, Skarstad K, Bloom B, Godal T, et al. (1982) Applications of flow cytometry on bacteria: cell cycle kinetics, drug effects, and quantitation of antibody binding. Cytometry 2: 249-257.

21. Haugland RP (1996) Handbook of fluorescent probes and research chemicals. $\left(\right.$ edn $\left.6^{\text {th }}\right)$ Eugene, Oreg: Molecular Probes Inc.

22. Mason DJ, Lloyd D (1997) Acridine orange as an indicator of bacteria susceptibility to gentamicin. FEMS Microbiol Lett 153: 199-204.

23. Comas J, Vives-Rego J (1997) Assessment of the effects of gramicidin formaldehyde, and surfactants on Escherichia coli by flow cytometry using nucleic acid and membrane potential dyes. Cytometry 29: 58-64.

24. Jepras RI, Carter J, Pearson SC, Paul FE, Wilkinson MJ (1995) Developmen of a robust flow cytometric assay for determining numbers of viable bacteria. Appl Environ Microbiol 61: 2696-2701.

25. Suller MTE, Stark JM, Lloyd D (1997) A flow cytometric study of antibiotic-induced damage and evaluation as a rapid antibiotic susceptibility test for methicillinresistant Staphylococcus aureus. J Antimicrob Chemother 40: 77-83.

26. Chopra L, Singh G, Kumar Jena K, Sahoo DK (2015) Sonorensin: A new bacteriocin with potential of an anti-biofilm agent and a food biopreservative. Sci Rep 5: 13412.

27. Weeks ME, Nebe von Caron G, James DC, Smales CM, Robinson GK (2006) Monitoring changes in nisin susceptibility of Listeria monocytogenes Scott $A$ as an indicator of growth phase using FACS. J Microbiol Methods 66: 43-55.

28. Jahn M, Seifert J, von Bergen M, Schmid A, Bühler B, et al. (2013) Subpopulation-proteomics in prokaryotic populations. Curr Opin Biotechno 24: 79-87.

29. Budde BB, Rasch M (2001) A comparative study on the use of flow cytometry and colony forming units for assessment of the antibacterial effect of bacteriocins. Int J Food Microbiol 63: 65-72.

30. Diaper JP, Edwards C (1994) Flow cytometric detection of viable bacteria in compost. FEMS Microbiol Ecol 14: 213-220.

31. Jacobsen CN, Rasmusson J, Jakobsen M (1997) Viability staining and flow cytometric detection of Listeria monocytogenes. J Microbiol Methods 28: 35-43.

32. Ayari S, Dussault D, Hayouni el A, Vu KD, Hamdi M, et al. (2012) Response of Bacillus cereus vegetative cells after exposure to repetitive sublethal radiation processing in combination with nisin. Food Microbiol 32: 361-370.

33. Dalmau M, Maier E, Mulet N, Viñas M, Benz R (2002) Bacterial membrane injuries induced by lactacin $\mathrm{F}$ and nisin. Int Microbiol 5: 73-80.

34. Ueckert JE, ter Steeg PF, Coote PJ (1998) Synergistic antibacterial action of heat in combination with nisin and magainin II amide. J Appl Microbiol 85: 487-494

35. Ueckert JE, Nebe von-Caron G, Bos AP, ter Steeg PF (1997) Flow cytometric analysis of Lactobacillus plantarum to monitor lag times, cell division and injury. Lett Appl Microbiol 25: 295-299.

36. Parish CR (1999) Fluorescent dyes for lymphocyte migration and proliferation studies. Immunol Cell Biol 77: 499-508.

37. Swarts AJ, Hastings JW, Roberts RF, von Holy A (1998) Flow cytometry demonstrates bacteriocin-induced injury to Listeria monocytogenes. Curr Microbiol 36: 266-270.
38. Pal G, Srivastava S (2014) Inhibitory effect of plantaricin peptides (PIn E/F and $\mathrm{J} / \mathrm{K}$ ) against Escherichia coli. World J Microbiol Biotechnol 30: 2829-2837.

39. Chen YC, Tsai TL, Ye XH, Lin TH (2015) Anti-proliferative effect on a colon adenocarcinoma cell line exerted by a membrane disrupting antimicrobial peptide KL15. Cancer Biol Ther 16: 1172-1183.

40. Tsai TL, Li AC, Chen YC, Liao YS, Lin TH (2015) Antimicrobial peptide m2163 or m2386 identified from Lactobacillus casei ATCC 334 can trigger apoptosis in the human colorectal cancer cell line SW480. Tumour Biol 36: 3775-3789.

41. Cooper GM (2000) The eukaryotic cell cycle. The Cell: A Molecular Approach (edn $\left.1^{\text {st }}\right)$, USA

42. Leuschner C, Hansel W (2004) Membrane disrupting lytic peptides for cancer treatments. Curr Pharm Des 10: 2299-2310.

43. Yates KR, Welsh J, Udegbunam NO, Greenman J, Maraveyas A, et al. (2012) Duramycin exhibits antiproliferative properties and induces apoptosis in tumour cells. Blood Coagul Fibrinolysis 23: 396-401.

44. Sand SL, Oppegård C, Ohara S, lijima T, Naderi S, et al. (2010) Plantaricin A a peptide pheromone produced by Lactobacillus plantarum, permeabilizes the cell membrane of both normal and cancerous lymphocytes and neuronal cells. Peptides 31: 1237-1244.

45. Sand SL, Haug TM, Nissen-Meyer J, Sand O (2007) The bacterial peptide pheromone plantaricin A permeabilizes cancerous, but not normal, rat pituitary cells and differentiates between the outer and inner membrane leaflet. J Membr Biol 216: 61-71.

46. Joo NE, Ritchie K, Kamarajan P, Miao D, Kapila YL (2012) Nisin, an apoptogenic bacteriocin and food preservative, attenuates HNSCC tumorigenesis via CHAC1. Cancer Med 1: 295-305.

47. Kamarajan P, Hayami T, Matte B, Liu Y, Danciu T, et al. (2015) Nisin ZP, a Bacteriocin and Food Preservative, Inhibits Head and Neck Cancer Tumorigenesis and Prolongs Survival. PLoS One 10: e0131008.

48. Gupta SM, Aranha CC, Bellare JR, Reddy KV (2009) Interaction of contraceptive antimicrobial peptide nisin with target cell membranes: implications for use as vaginal microbicide. Contraception 80: 299-307.

49. Hetz C, Bono MR, Barros LF, Lagos R (2002) Microcin E492, a channel-forming bacteriocin from Klebsiella pneumoniae, induces apoptosis in some human cell lines. Proc Natl Acad Sci U S A 99: 2696-2701.

50. Chumchalová J, Smarda J (2003) Human tumor cells are selectively inhibited by colicins. Folia Microbiol (Praha) 48: 111-115.

51. Lequerica JL, O'Connor JE, Such L, Alberola A, Meseguer I, et al. (2006) A halocin acting on $\mathrm{Na}+/ \mathrm{H}+$ exchanger of haloarchaea as a new type of inhibitor in NHE of mammals. J Physiol Biochem 62: 253-262.

52. Torreblanca M, Meseguer I, Venosa A (1994) Production of halocin is practically universal feature of archaeal halophilic rods. Letters in Appl Microbiol 19: $201-205$

53. Meseguer I, Torreblanca M, Konishi T (1995) Specific inhibition of the halobacterial $\mathrm{Na}+/ \mathrm{H}+$ antiporter by halocin $\mathrm{H} 6$. J Biol Chem 270: 6450-6455.

54. Davey HM, Kell DB (1996) Flow cytometry and cell sorting of heterogeneous microbial populations: the importance of single-cell analyses. Microbiol Rev 60: 641-696.

55. Lloyd D (1993) Flow cytometry in microbiology. Springer-Verlag. London, United Kingdom

56. Siegman-Igra Y, Kulka T, Schwartz D, Konforti N (1993) The significance of polymicrobial growth in urine: contamination or true infection. Scand J Infect Dis 25: 85-91.

57. Gottfredsson M, Erlendsdottir H, Sigfusson A, Gudmundsson S (1998) Characteristics and dynamics of bacterial populations during postantibiotic effect determined by flow cytometry. Antimicrob Agents Chemother 42: 10051011

58. Suller MT, Lloyd D (1998) Flow cytometric assessment of the postantibiotic effect of methicillin on Staphylococcus aureus. Antimicrob Agents Chemother 42: 1195-1199.

59. Mason DJ, Power EG, Talsania H, Phillips I, Gant VA (1995) Antibacterial action of ciprofloxacin. Antimicrob Agents Chemother 39: 2752-2758.

60. Roth BL, Poot M, Yue ST, Millard PJ (1997) Bacterial viability and antibiotic 
Citation: Mathur H, Rea MC, Fallico V, Cotter PD, Hill C et al. (2016) Flow Cytometry as a Tool to Study the Effects of Bacteriocins on Prokaryotic and Eukaryotic Cells. J Mol Biomarkers Diagn S8:013. doi:10.4172/2155-9929.S8-013

Page 8 of 6

susceptibility testing with SYTOX green nucleic acid stain. Appl Environ Microbiol 63: 2421-2431.

61. Mortimer FC, Mason DJ, Gant VA (2000) Flow cytometric monitoring of antibiotic-induced injury in Escherichia coli using cell-impermeant fluorescent probes. Antimicrob Agents Chemother 44: 676-681.

62. Novo DJ, Perlmutter NG, Hunt RH, Shapiro HM (2000) Multiparameter flow cytometric analysis of antibiotic effects on membrane potential, membrane permeability, and bacterial counts of Staphylococcus aureus and Micrococcus luteus. Antimicrob Agents Chemother 44: 827-834.

63. Wickens HJ, Pinney RJ, Mason DJ, Gant VA (2000) Flow cytometric investigation of filamentation, membrane patency, and membrane potential in Escherichia coli following ciprofloxacin exposure. Antimicrob Agents Chemother 44: 682-687.

64. Laflamme C, Lavigne S, Ho J, Duchaine C (2004) Assessment of bacterial endospore viability with fluorescent dyes. J Appl Microbiol 96: 684-692.

65. Lehtinen J, Nuutila J, Lilius E (2004) Green fluorescent protein-propidium iodide (GFP-PI) based assay for flow cytometric measurement of bacterial viability. Cytometry A 60: 165-172.

66. Papadimitriou K, Pratsinis H, Nebe-von-Caron G, Kletsas D, Tsakalidou E (2006) Rapid assessment of the physiological status of Streptococcus macedonicus by flow cytometry and fluorescence probes. Int J Food Microbio 111: 197-205.

67. Nebe-von-Caron G, Stephens PJ, Hewitt CJ, Powell JR, Badley RA (2000) Analysis of bacterial function by multi-colour fluorescence flow cytometry and single cell sorting. J Microbiol Methods 42: 97-114.

68. Oliver JD (2005) The viable but nonculturable state in bacteria. J Microbiol 43 Spec No: 93-100.

69. Pyle BH, Broadaway SC, McFeters GA (1999) Sensitive detection of Escherichia coli $0157: \mathrm{H} 7$ in food and water by immunomagnetic separation and solid-phase laser cytometry. Appl Environ Microbiol 65: 1966-1972.

70. Khan MM, Pyle BH, Camper AK (2010) Specific and rapid enumeration of viable but nonculturable and viable-culturable gram-negative bacteria by using flow cytometry. Appl Environ Microbiol 76: 5088-5096.

71. Garcia-Betancur JC, Yepes A, Schneider J, Lopez D (2012) Single-cell analysis of Bacillus subtilis biofilms using fluorescence microscopy and flow cytometry. Journal of Visualized Experiments 60: e3796.

72. Vlamakis H, Aguilar C, Losick R, Kolter R (2008) Control of cell fate by the formation of an architecturally complex bacterial community. Genes Dev 22 945-953.

73. Branda SS, Chu F, Kearns DB, Losick R, Kolter R (2006) A major protein component of the Bacillus subtilis biofilm matrix. Mol Microbiol 59: 1229-1238.

74. Mason DJ, Gant VA (1995) The application of flow cytometry to the estimation of bacterial antibiotic susceptibility. J Antimicrob Chemother 36: 441-443.

75. Matsuyama T (1984) Staining of living bacteria with rhodamine 123. FEMS Microbiol Lett. 21: 153-157.

76. Diaper JP, Tither K, Edwards C (1992) Rapid assessment of bacterial viability by flow cytometry. Appl Microbiol Biotechnol 38: 268-272.

77. Eyler YL, Lantz LM, Lewis AM Jr. (1994) Flow cytometric detection of DNA tumor virus nuclear oncogene products in unfixed cells: saponin FACS of viral oncogene products. J Virol Methods 46: 23-27.

78. Jernaes MW, Steen HB (1994) Staining of Escherichia coli for flow cytometry influx and efflux of ethidium bromide. Cytometry 17: 302-309.

79. Midgley M (1986) The phosphonium ion efflux system of Escherichia coli relationship to the ethidium efflux system and energetic studies. J Gen Microbiol 132: 3187-3193.

80. Gou J, Lee HY, Ahn J (2010) Inactivation kinetics and virulence potential of Salmonella Typhimurium and Listeria monocytogenes treated by combined high pressure and nisin. J Food Prot 73: 2203-2210.

81. Ratinaud MH, Revidon S (1996) A flow cytometric method to assess functiona state of the Listeria membrane. J Microbiol Methods 25: 71-77.
This article was originally published in a special issue, Potential Biomarkers and Therapeutic Targets in Cancer Stem Cells handled by Editor(s). Dr. Murielle Mimeault, University of Nebraska Medical Center, USA 